\title{
FIB MICRO-PILLAR SAMPLING TECHNIQUE FOR 3D STEM OBSERVATION AND ITS APPLICATION
}

T. Yaguchi*, M.Konno*, T. Kamino*, T. Hashimoto**, T. Onishi**and K.Umemura***, K.Asayama****

*Hitachi Science Systems Ltd., 11-1 Ishikawa-cho, Hitachinaka, Ibaraki, 312-0057 Japan

**Hitachi High-Technologies Corp., 882 Ichige, Hitachinaka, Ibaraki, 312-8504 Japan

***Central Research laboratory Hitachi Ltd. 1-180 Higashikoigakubo, Kokubunji, Tokyo, 185-8601 Japan

****Semiconductor and Integrated Circuits Division, Hitachi Ltd., 20-1 Josuihon-cho 5, Kodaira, Tokyo 187-8588 Japan

A novel method allowing observation of three-dimensional structures of a specific site of materials has been developed. A dedicated focused ion beam (FIB) system equipped with a micro-sampling unit is used for specimen preparation and a dedicated scanning transmission electron microscope (STEM) equipped with a $200 \mathrm{kV}$ cold field emitter is used for the observation. A new FIB-STEM compatible specimen holder equipped with a specimen stage rotation mechanism and conical specimen stage has been also developed for the method. In this holder, specimen is mounted on the flattened top of the cone. The rotation mechanism of the holder allows $360^{\circ}$ turning of the conic stage around the ion beam axis of the FIB system and $360^{\circ}$ turning perpendicular to the electron beam in the STEM. In this method, a piece of sample in a shape of pillar is extracted from the site to be characterized by the FIB micro-sampling method ${ }^{1-2)}$. The extracted micro-pillar sample is transferred and mounted on the conical stage. The sample is shaped into a pillar of 2-5 microns square and 10 to 15 microns long, and then transferred to the STEM for high angle annular dark field (HAADF) STEM, bright field STEM and secondary electron microscopy (SEM) images observation at $200 \mathrm{kV}$ to obtain three dimensional structure and cross sectional structural of the area to be characterized. Geometry of the sample and sample stage allows observation of the sample from unlimited angles. Thus, specific site is localized for further FIB milling whenever it is required. . Figure 1 shows the procedure of the method. Example of STEM(a) and SEM images(b) observation of $2 \mu \mathrm{m} \times 2 \mu \mathrm{m}$-micro-pillar sample of a Si device is shown in Figure 2. Inside and cross sectional structures of the $\mathrm{Si}$ device are clearly observed. Example of EDX mapping image observation is shown in Figure3. Thickness of the sample is 2 micron. Ti-K and Al-K images are clearly observed.

\section{References}

1) T.Onishi, H.Koike, T.Ishitani, S.Tomimatsu, K.Umemura and T.Kamino: Proc. 25 th Int. Symp. for Testing and Failure Analysis (1999) 449-453

2) T.Kamino, T.Yaguchi, Y.Kuroda, T.Hashimoto, T.Onishi, T.Ishitani, K.umemura and K.Asayama, Proc. Microsc. Microanal. 8 (Suppl .2) (2002) 48-49 


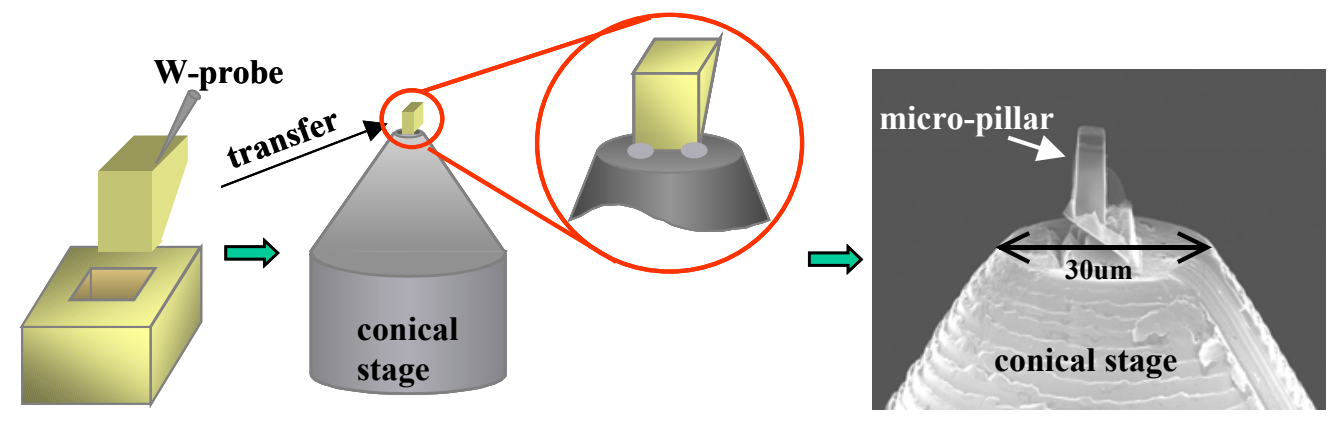

micro-sampling

trimming

Figure 1. Procedure for preparing micro-pillar sample by FIB
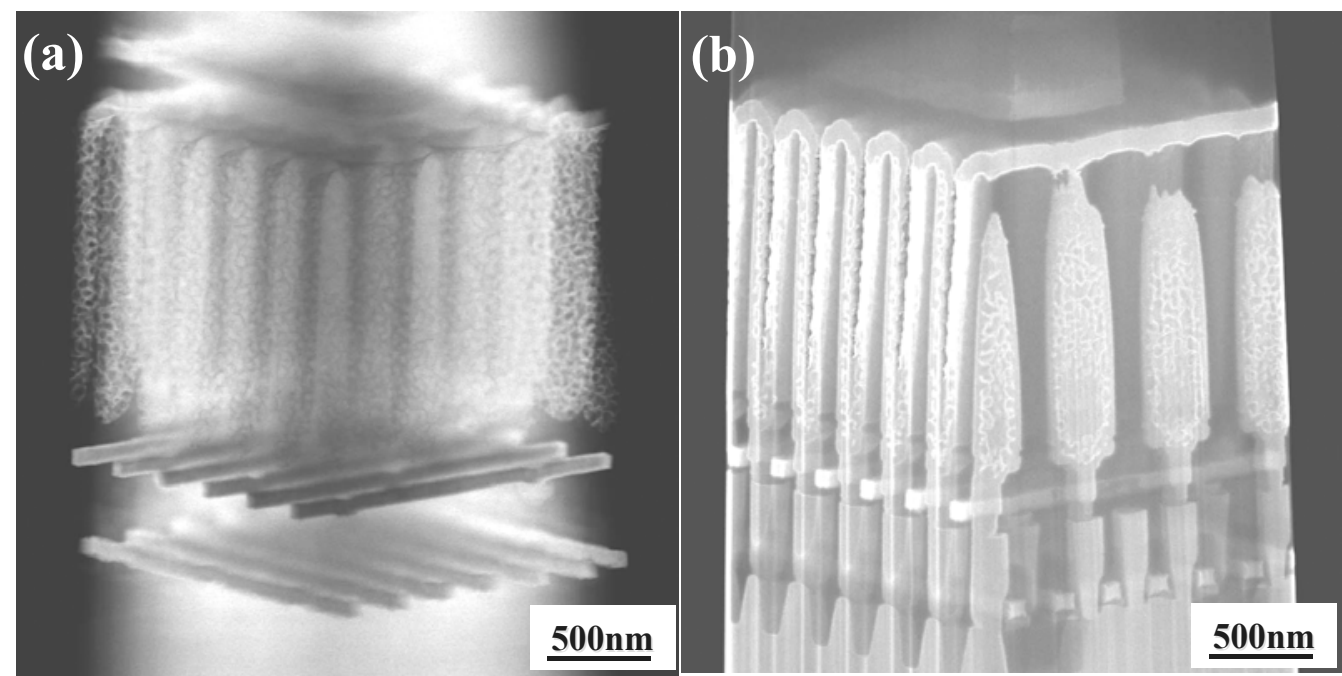

Figure 2. STEM(a) and SEM(b) images of $2 \mu \mathrm{m} \times 2 \mu \mathrm{m}$ pillar sample of $\mathrm{Si}$ device observed from the same direction.
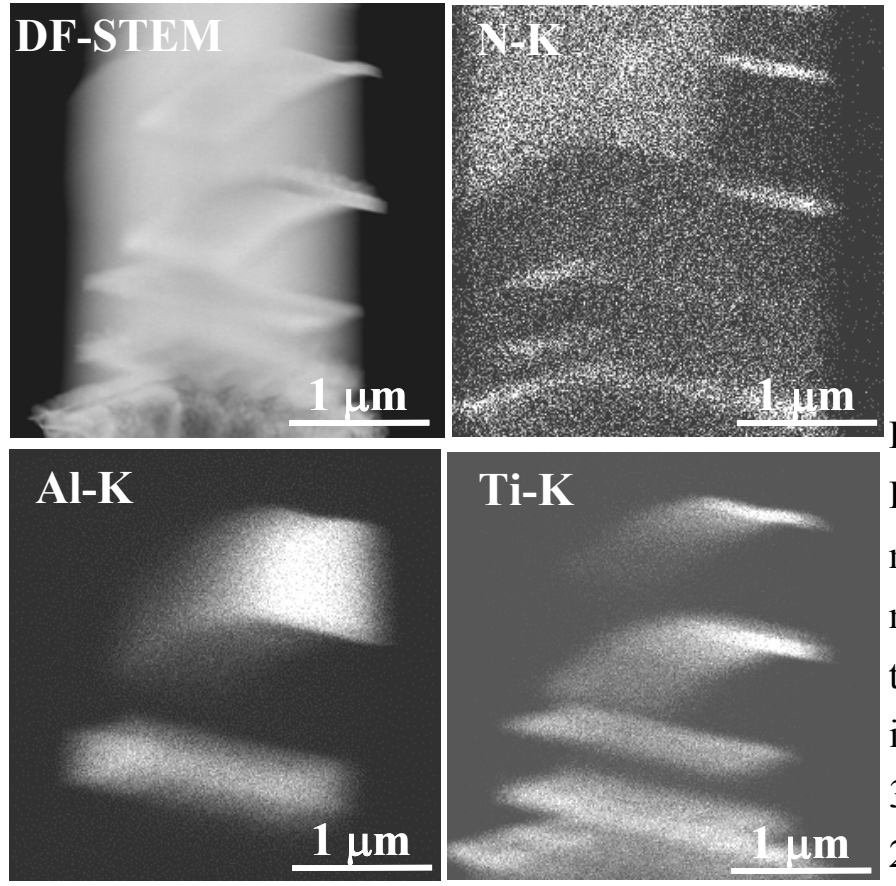

Figure 3.

DF STEM and the elemental mapping images of the micro-pillar sample with the thickness of 2 micron. The integration time was 30minutes. Pixel sizes are $256 \times 256$. 